\title{
Correlation between spectral domain optical coherence tomography findings and visual outcomes in central retinal vein occlusion
}

This article was published in the following Dove Press journal:

Clinical Ophthalmology

| March 201 |

Number of times this article has been viewed

\author{
Verônica Castro Lima' \\ Ling Yeung ${ }^{1,2}$ \\ Leonardo C Castro ${ }^{3}$ \\ Gennady Landa' \\ Richard B Rosen ${ }^{1,4}$ \\ 'Retina Service, Department of \\ Ophthalmology, The New York Eye \\ and Ear Infirmary, New York, NY, USA; \\ ${ }^{2}$ Department of Ophthalmology, \\ Chang Gung Memorial Hospital, \\ Keelung, Taiwan; ${ }^{3} \mathrm{New}$ England Eye \\ Center, Tufts Medical Center, Boston, \\ MA, USA; ${ }^{4}$ The New York Medical \\ College, Valhalla, NY, USA
}

Purpose: To investigate the relationship between spectral domain optical coherence tomography (SD-OCT) findings and visual outcomes following resolution of macular edema in central retinal vein occlusion (CRVO).

Methods: Patients with recent onset CRVO who had undergone SD-OCT and fluorescein angiography (FA) exams on the day of initial presentation were included. All patients had resolution of macular edema in SD-OCT images at the end of follow-up, and they were separated into two groups according to final visual acuity: group $1(\leq 20 / 200)$ and group $2(>20 / 200)$. SD-OCT scans and FA studies were analyzed in a masked fashion. Macular perfusion status by FA was categorized according to presence or absence of macular ischemia.

Results: A total of 22 eyes from 22 patients [mean age $53.0 \pm 15.0$ (standard deviation)] were included. Mean follow up period was $14.6 \pm 8.1$ (standard deviation) months. Group 1 (10 eyes) had significantly higher rates of residual intraretinal fluid, loss of foveal inner segment/outer segment (IS/OS) junction line and loss of inner retinal layers in late stage SD-OCT images $(P=0.027)$ when compared with group 2 (12 eyes). Loss of foveal IS/OS junction line (odds ratio $[\mathrm{OR}]=13.826 ; P=0.098)$ and loss of inner retinal layers $(\mathrm{OR}=38.908 ; P=0.013)$ in late stage SD-OCT images were correlated with poorer final visual outcomes. Macular ischemia by FA correlated with thinner central subfield thickness $(r=-0.54, P=0.021)$ and loss of inner retinal layers $(r=0.47, P=0.031)$ in early stage SD-OCT images; and presence of residual intraretinal fluid $(r=0.61, P=0.003)$ and loss of inner retinal layers $(r=0.71, P<0.001)$ in late stage SD-OCT images.

Conclusion: Loss of foveal IS/OS junction line and inner retinal layers on SD-OCT significantly correlated with poorer visual outcomes in CRVO patients.

Keywords: macular edema, inner retinal layers, macular ischemia

\section{Introduction}

Central retinal vein occlusion (CRVO) is a frequent retinal vascular disorder, often associated with severe visual loss. ${ }^{1,2}$ Macular edema is the most common vision-threatening complication associated with CRVO. Various options for treatment of macular edema secondary to CRVO have been studied, such as laser photocoagulation, ${ }^{3}$ radial optic neurotomy, ${ }^{4}$ and intravitreal injection of various agents including tissue plasminogen activator, ${ }^{5}$ triamcinolone acetonide, ${ }^{6}$ or anti-vascular endothelial growth factor agents. ${ }^{7,8}$ However, there is no proven remedy for visual loss due to CRVO. ${ }^{9}$ Despite the observation that resolution of macular edema often leads to visual improvement, some patients suffer persistent poor vision following complete resolution of the macular edema. ${ }^{10}$
Correspondence: Richard B Rosen Department of Ophthalmology, The New York Eye and Ear Infirmary, 310 East 14th Street, New York, NY 10003, USA

Email rrosen@nyee.edu 
Optical coherence tomography (OCT) is a noncontact, noninvasive method that allows quantitative determination of retinal thickness and volume. It provides cross-sectional slice images of the retina and useful information on vitreoretinal interface changes associated with various posterior segment disorders. ${ }^{11-13}$ Spectral domain OCT (SD-OCT) is the latest generation of the technology which provides images of higher axial resolution and reduced motion artifacts. ${ }^{14-16}$ It permits accurate identification of individual retinal layers with nearhistological detail and provides significant clinicopathological information in many retinal conditions. ${ }^{17,18} \mathrm{~A}$ number of recent OCT studies have identified that continuity of the photoreceptor inner segment/outer segment (IS/OS) junction line in the fovea is a hallmark of foveal photoreceptor integrity, which is critical to visual outcome in eyes with disorders such as branch retinal vein occlusion (BRVO) ${ }^{19-21}$ and CRVO. ${ }^{10}$ However, there is scant information in the literature unraveling the relationship between specific retinal structural changes recorded in SD-OCT images and visual outcomes in eyes with CRVO.

In the current study, we investigated whether early and late SD-OCT findings, such as loss of inner retinal layers and foveal IS/OS junction status, correlated with visual acuity following resolution of macular edema in patients with CRVO. Additionally, we looked at the relationship between macular perfusion revealed by fluorescein angiography (FA) and its association with those SD-OCT findings.

\section{Methods}

A retrospective chart review was conducted surveying the imaging database for patients with CRVO who underwent both SD-OCT and FA exam during initial clinical evaluation at the Retina Service of The New York Eye and Ear Infirmary, between September 2006 and September 2008. The study was approved by the Institutional Review Board of the New York Eye and Ear Infirmary.

Patients with CRVO, who presented within 1 month of onset of symptoms, were included in the study. Patients had to have at least 6 months of follow-up, and resolution of macular edema on clinical exam, and by the SD-OCT (central foveal thickness $<250 \mu \mathrm{m}$ ) at the end of this period. Eyes with poor quality SD-OCT scans or FA images, or patients with macular edema caused by other etiologies such as diabetic retinopathy, postoperative or inflammatory cystoid macular edema, were excluded. In patients with bilateral CRVO, one eye was selected randomly to be included. Medical records of all eligible patients were reviewed, and demographics, systemic comorbidities, and treatments performed were recorded. Best corrected Snellen visual acuity measurements recorded during the initial visit and following resolution of macular edema were collected. Initial best corrected visual acuity (BCVA) was defined as that recorded during initial presentation to our service. Final BCVA was taken as the one recorded at follow-up examination after resolution of macular edema. Based upon the final BCVA, patients were separated into two groups: group 1 $(\mathrm{BCVA} \leq 20 / 200)$ and group $2(\mathrm{BCVA}>20 / 200)$.

The SD-OCT (Spectral OCT/SLO, OPKO/OTI, Miami, FL) device used in this study had transverse and axial resolutions of 20 and 5-6 $\mu \mathrm{m}$, respectively. Individual cross-section images covered a field of $29^{\circ}$ and came with a paired scanning laser ophthalmoscopy (SLO) image displaying the origin of the slice. Images considered acceptable in quality were evaluated by two masked retina specialists for evidence of predefined macular abnormalities and central retinal thickness. Integrity of the foveal photoreceptor layer was evaluated using high quality raw grayscale SD-OCT scans to determine the condition of the IS/OS junction line. Eyes were classified as having loss of the IS/OS junction when this line was totally absent in the fovea. In all patients, SD-OCT scans to evaluate macular thickness and to assess effectiveness of treatment during follow-up were performed until they met the satisfaction of the operator, an ophthalmologist with extensive imaging experience. Early stage and late stage SD-OCT findings refer to those macular changes recorded in the SD-OCT images taken during the initial visit and following resolution of macular edema, respectively.

FA assessment of macular ischemia was interpreted by one masked observer, and studies were separated into two categories, according to the presence or absence of macular ischemia. The patients with foveal avascular zones (FAZs) $\geq 1000 \mu \mathrm{m}$, or broken perifoveal capillary rings at the borders of the FAZ or distinct areas of capillary nonperfusion within one disk diameter of the foveal center, as judged in FA transit phase frames, were regarded as having macular ischemia. $^{22}$

\section{Statistical analysis}

Chi-square analysis was used for categorical data, and the independent sample student $t$-test was used for continuous data to determine whether significant differences appeared between groups 1 and 2. Fisher's exact test was used when one or more of the cells in chi-square analysis had expected values below 5. All clinical manifestations, SD-OCT findings, and presence of macular ischemia on FA 
were submitted for analysis. A two-tailed $P$-value of $<0.05$ was considered statistically significant. Logistic regression model was used to evaluate the risk factors for poorer visual outcome (BCVA $\leq 20 / 200)$. Potential risk factors including age, initial visual acuity, and SD-OCT findings that showed differences which were statistically significant between groups were analyzed by backward stepwise regression. Factors with $P$-value $>0.05$ were removed from the equation. Spearman's correlation coefficient was used to analyze the relationship between SD-OCT findings and macular ischemia by FA. All data were analyzed using SPSS Program Package Version 15.0 (SPSS Inc., Chicago, IL). Logistic regression power was calculated using PASS 2008 (NCSS, Kaysville, UT) software.

\section{Results}

Twenty-two patients (22 eyes) diagnosed with CRVO were included [ 9 female and 13 male; mean age of $53.0 \pm 15.0$ (standard deviation) years]. Co-morbidities included systemic arterial hypertension (45\%), dyslipidemia (23\%), type 2 diabetes mellitus (9\%), and acquired immunodeficiency syndrome (4.5\%). Treatment modalities used for macular edema included intravitreal injections of bevacizumab in $10(45 \%)$ eyes and triamcinolone acetonide in four (18\%) eyes. Multiple injections had been administered if required for resolution of the macular edema. Five eyes in group 2 had received no treatment for macular edema due to good vision at presentation. Peripheral panretinal photocoagulation was performed in $6(27 \%)$ eyes. Two eyes developed neovascular glaucoma despite laser treatment. The mean follow-up period for all patients was $14.6 \pm 8.1$ (standard deviation) months (range, 7-42 months). Foveal thickness and central subfield thickness could not be determined on early stage SD-OCT in three patients due to severe macular edema beyond the range of the instrument (2.0 mm maximum depth). FA images of one patient were missing. This patient was excluded from the analysis of the relationship between the macular perfusion status and SD-OCT findings.

Among the 22 eyes included, 10 (45\%) eyes had final BCVA $\leq 20 / 200$ (group 1), and 12 (55\%) eyes had final BCVA $>20 / 200$ (group 2). Table 1 summarizes clinical characteristics, SD-OCT findings, and macular perfusion status for each group. There were no statistically significant differences in age, gender, and initial visual acuity between groups. There were also no significant differences in foveal thickness and central subfield thickness between the two groups before and after resolution of macular edema. The main findings analyzed in the SD-OCT scans were retention of hyperreflectivity of the inner layers, presence of intraretinal fluid, presence of serous retinal detachment, loss of foveal IS/OS junction, and loss of inner retinal layers (Figures 1 and 2).

Statistical analysis showed that eyes in group 1 had higher rates of residual intraretinal fluid $(P=0.027)$, loss of foveal IS/OS junction $(P=0.020)$ and loss of inner retinal layers $(P=0.002)$ in late stage SD-OCT images compared with those in group 2. To determine the risk factors for poorer visual outcome $(\mathrm{BCVA} \leq 20 / 200)$, the SD-OCT findings described above, along with age and initial visual acuity, were analyzed using a logistic regression model. Among all findings analyzed, loss of foveal IS/OS junction line (odds ratio $[\mathrm{OR}]=13.826 ; P=0.098 ; 95 \%$ confidence interval $[\mathrm{CI}]: 0.616-310.321)$ and loss of inner retinal layers $(\mathrm{OR}=38.908 ; P=0.013$; 95\% CI: 2.169-698.087) in late stage SD-OCT images correlated with poorer visual outcomes. The power of this logistic regression model was 0.703 .

Group 1 had more eyes with macular ischemia by FA at initial presentation when compared with group $2(P=0.009)$. Table 2 summarizes the SD-OCT findings in eyes with and without macular ischemia by FA. Macular ischemia by FA showed significant correlation with the following SD-OCT findings: thinner central subfield thickness $(r=-0.54$, $P=0.021)$ and loss of inner retinal layers $(r=0.47, P=0.031)$ in early stage SD-OCT images; and presence of residual intraretinal fluid $(r=0.61, P=0.003)$ and loss of inner retinal layers $(r=0.71, P<0.001)$ in late stage SD-OCT images.

\section{Discussion}

In this study we found that loss of foveal IS/OS junction line and absence of inner retinal layers in late stage SD-OCT images were significantly correlated with poor visual outcomes in patients with CRVO. Additionally, loss of inner retinal layers in late stage SD-OCT images correlated significantly with macular ischemia diagnosed in early FA studies.

Currently, agents such as intravitreal triamcinolone acetonide or bevacizumab have been shown to be effective for reducing the macular edema associated with CRVO. ${ }^{6-8}$ In most cases, reduction of macular edema is associated with visual acuity improvement. However, only some patients are able to achieve full recovery of vision following resolution of macular edema, and others experience only limited visual improvement despite "successful" treatment. In fact, the relationship between central retinal thickness and visual acuity has not been well established 
Table I Demographic and clinical characteristics of each group of patients with central retinal vein occlusion

\begin{tabular}{|c|c|c|c|}
\hline & $\begin{array}{l}\text { Group I } \\
\text { (final VA } \leq 20 / 200)\end{array}$ & $\begin{array}{l}\text { Group } 2 \\
\text { (final VA }>20 / 200)\end{array}$ & $P$ value ${ }^{a}$ \\
\hline Number of patients & 10 & 12 & \\
\hline Age (years, mean $\pm S D$ ) & $59.6 \pm 12.8$ & $47.6 \pm 14.9$ & 0.059 \\
\hline Gender (female:male) & $3: 7$ & $6: 6$ & 0.415 \\
\hline \multicolumn{4}{|l|}{ Systemic diseases } \\
\hline Hypertension & 6 & 5 & 0.392 \\
\hline Dyslipidemia & 2 & 0 & 0.195 \\
\hline Diabetes mellitus & 2 & 0 & 0.195 \\
\hline Duration of CRVO [median (range)] & I month ( 2 days to 3 months) & 2 weeks ( 0 day to I month) & \\
\hline \multicolumn{4}{|l|}{ Initial VA } \\
\hline$\leq 20 / 200$ & 9 & 7 & \multirow{2}{*}{0.162} \\
\hline$>20 / 200$ & I & 5 & \\
\hline \multicolumn{4}{|l|}{ Early stage SD-OCT findings } \\
\hline Foveal thickness $(\mu \mathrm{m}$, mean $\pm S D)$ & $395 \pm 183$ & $488 \pm 179$ & 0.281 \\
\hline Central subfield thickness $(\mu \mathrm{m}$, mean $\pm S D)$ & $405 \pm 136$ & $473 \pm 107$ & 0.245 \\
\hline Hyperreflectivity inner layer & 9 & 12 & 0.455 \\
\hline Intraretinal fluid & 10 & 12 & - \\
\hline Serous retinal detachment & 5 & 5 & 1.000 \\
\hline Loss of foveal IS/OS junction & 2 & 3 & 1.000 \\
\hline Loss of inner retinal layers & 3 & 0 & 0.078 \\
\hline \multicolumn{4}{|l|}{ Late stage SD-OCT findings } \\
\hline Foveal thickness (mean $\pm S D$ ) & $130 \pm 97$ & $155 \pm 102$ & 0.574 \\
\hline Central subfield thickness (mean $\pm S D$ ) & $182 \pm 76$ & $207 \pm 101$ & 0.524 \\
\hline Hyperreflectivity inner layer & 2 & 2 & 1.000 \\
\hline Residual intraretinal fluid & 7 & 2 & $0.027^{\mathrm{b}}$ \\
\hline Serous retinal detachment & 0 & 0 & - \\
\hline Loss of foveal IS/OS junction & 6 & I & $0.020^{\mathrm{b}}$ \\
\hline Loss of inner retinal layers & 8 & I & $0.002^{\mathrm{b}}$ \\
\hline Macular ischemia on FA & 7 & 2 & $0.009^{b}$ \\
\hline
\end{tabular}

Notes: ${ }^{a}$ Chi-square analysis or Fisher's exact test was used for categorical data, and independent sample student $t$-test was used for continuous data. ${ }^{\mathrm{A}} \mathrm{A}$ two-tailed $P$ value of $<0.05$ was considered statistically significant.

Abbreviations: FA, fluorescein angiography; SD, standard deviation; SD-OCT, spectral domain optical coherence tomography; VA, visual acuity.

in cases of CRVO. Scott et $\mathrm{l}^{23}$ demonstrated that the correlation between OCT-measured center point thickness, and visual acuity letter score was statistically significant, but this relationship was, at best, modest in patients with macular edema associated with retinal vein occlusions.
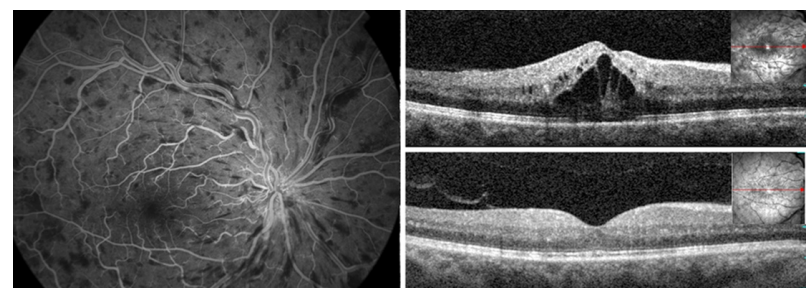

Figure I Fluorescein angiography (20.9 seconds) of one patient with recent onset central retinal vein occlusion in the right eye showing no evidence of ischemia in the macula (left). Gray-scale spectral domain optical coherence tomography (SD-OCT) horizontal scan at the initial visit (upper right) demonstrates prominent macular edema with cystoid spaces and serous retinal detachment under the fovea. The Snellen best corrected visual acuity was 20/200 at this point. This patient was treated with multiple intravitreal injections of bevacizumab. Gray-scale SD-OCT horizontal scan at the final visit (lower right) shows that macular edema has resolved completely. The inner segment/outer segment (IS/OS) junction can still be identified in the fovea, and there is a complete preservation of the inner retinal architecture. Visual acuity improved to $20 / 25$ at final visit.
In our study, there was a trend, though not significant statistically, for patients with poorer visual outcomes to have had thinner mean foveal thickness and central subfield thickness in both early and late stage SD-OCT imaging when compared with patients achieving better
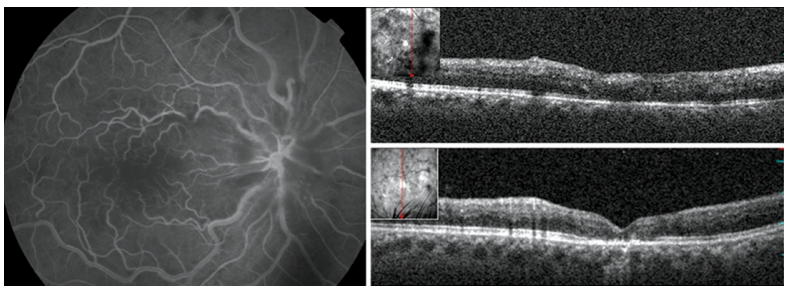

Figure 2 Fluorescein angiography (35.I seconds) of another patient with recent onset central retinal vein occlusion in the right eye revealing ischemia in the macular area (left). Gray-scale spectral domain optical coherence tomography (SD-OCT) vertical scan at the initial visit (upper right) demonstrates the presence of a discrete amount of subretinal fluid under the fovea and loss of inner retinal layers. Visual acuity was counting fingers at this point. This patient was treated with an intravitreal injection of bevacizumab and panretinal photocoagulation. Gray-scale SD-OCT vertical scan at the final visit (lower right) shows resolution of subretinal fluid under the fovea. The inner segment/outer segment (IS/OS) cannot be identified in the fovea and there is loss of the normal inner retina architecture. Visual acuity was $20 / 400$ at final visit. 
Table 2 Comparison between eyes with and without macular ischemia on FA

\begin{tabular}{|c|c|c|c|}
\hline & \multicolumn{3}{|c|}{ Macular ischemia on FA } \\
\hline & Absent (\%) & Present (\%) & $P$ value ${ }^{a}$ \\
\hline Number of eyes & 12 & 9 & \\
\hline \multicolumn{4}{|l|}{ Early stage SD-OCT findings } \\
\hline Foveal thickness ( $\mu \mathrm{m}$, mean $\pm S D)$ & $527 \pm 195$ & $362 \pm 122$ & 0.054 \\
\hline Central subfield thickness $(\mu \mathrm{m}$, mean $\pm \mathrm{SD})$ & $500 \pm 115$ & $383 \pm 105$ & $0.04 I^{b}$ \\
\hline Hyperreflectivity inner layer & $12(100)$ & $8(89)$ & 0.258 \\
\hline Intraretinal fluid & $12(100)$ & $9(100)$ & - \\
\hline Serous retinal detachment & $7(58)$ & $2(22)$ & 0.108 \\
\hline Loss of foveal IS/OS junction & $3(25)$ & $2(22)$ & 0.890 \\
\hline Loss of inner retinal layers & $0(0)$ & $3(33)$ & $0.031^{b}$ \\
\hline \multicolumn{4}{|l|}{ Late stage SD-OCT findings } \\
\hline Foveal thickness $(\mu \mathrm{m}$, mean $\pm S D)$ & $148 \pm 109$ & $148 \pm 87$ & 0.993 \\
\hline Central subfield thickness $(\mu \mathrm{m}$, mean $\pm S D)$ & $205 \pm 193$ & $|93 \pm 7|$ & 0.778 \\
\hline Hyperreflectivity inner layer & $2(17)$ & $2(22)$ & 0.763 \\
\hline Residual intraretinal fluid & $2(17)$ & $7(78)$ & $0.003^{b}$ \\
\hline Serous retinal detachment & $0(0)$ & $0(0)$ & - \\
\hline Loss of foveal IS/OS junction & $3(25)$ & $4(44)$ & 0.375 \\
\hline Loss of inner retinal layers & I (8) & $7(78)$ & $<0.00 \mathrm{I}^{\mathrm{b}}$ \\
\hline
\end{tabular}

visual outcomes. More importantly, macular ischemia by FA was significantly correlated with thinner central subfield thickness in early stage SD-OCT images. This appeared to go along with atrophic changes in the retina of those patients due to the macular ischemia. Thus, macular thickness alone, in this study, did not appear to be a good predictor of visual outcome in patients with CRVO.

According to histologic reports of eyes with retinal vein occlusions, edema appears at the level of the outer nuclear layer and is associated with liquefaction necrosis which may result in photoreceptor loss and contribute to foveal dysfunction. ${ }^{24}$ Previous studies using earlier third-generation time domain OCT devices found that IS/OS junction integrity at the fovea was closely associated with visual acuity in eyes with BRVO ${ }^{19-21}$ and CRVO. ${ }^{10}$ Most of these studies evaluated the status of the IS/OS junction line following resolution of macular edema as a possible hallmark of integrity of the photoreceptors layer and predictor of function. In the current study, using a faster and higher resolution SD-OCT device, which provided better visualization of the IS/OS junction, we were additionally able to resolve concurrent loss of inner retinal layers, which correlated with poor visual outcomes in these CRVO eyes.

The ability of SD-OCT technology to differentiate changes in individual retinal layers allowed us to correlate poorer visual outcome specifically to loss of inner retinal layers. This goes along with the pathophysiology of CRVO which has its greatest impact on the circulation of the inner retinal layers. Histopathological studies of areas of capillary nonperfusion following retinal vein occlusion have revealed the presence of inner ischemic atrophy with loss of inner plexiform and inner nuclear layers. ${ }^{25-27}$ These are consistent with the SD-OCT appearance of inner retinal architecture disappearance in patients with more severe macular ischemia and limited visual recovery. It also agrees with the statistically significant correlation between the finding of macular ischemia by FA and the loss of inner retinal layers on SD-OCT.

It is still unclear at what point these retinal structural changes occur in the course of CRVO. We were unable to demonstrate any difference in early stage SD-OCT image findings between groups 1 and 2. This suggests that some time interval is required before structural changes such as loss of IS/OS junction line and loss of inner retinal layers occur. In addition, during acute stages of the disease, visualization of the retinal morphology is often impaired due to scattering blur produced by intraretinal fluid and shadow artifacts caused by hemorrhage. These limitations mirror the difficulties diagnosing macular ischemia by FA in very hemorrhagic occlusions. Given the complementary nature of the information received from the two studies, its clear that SD-OCT and FA exams together provide a more complete clinical assessment for management of patients with CRVO. 
In previous reports, initial visual acuity was often found to be the most reliable prognostic factor for predicting visual prognosis in eyes with CRVO. ${ }^{1,2,28}$ It is, however, occasionally misleading due to transient macular edema or intraretinal hemorrhage. In our study, we similarly found that patients with poorer initial visual acuity were more likely to have worse longterm visual outcomes, but the correlation was not statistically significant, perhaps due to our small sample size.

Using multiple regression analysis, loss of the foveal IS/OS junction line and loss of inner retinal layers came up as risk factors for poorer visual outcome. Macular ischemia was not included in this particular regression model because it was highly correlated with loss of inner retinal layers. Given that our purpose was to focus on SD-OCT abnormal findings in relation to CRVO visual outcomes, we chose inner retinal layer loss instead of macular ischemia by FA to include in the regression analysis.

There were certain limitations to our study. First, it is based upon a relatively small sample size. Second, it is a retrospective chart review. And finally, it included patients treated with a variety of treatment modalities including intravitreal injections of bevacizumab and triamcinolone and panretinal photocoagulation. A small number of patients ( 5 eyes) did not receive any kind of treatment, and all of them were in group 2. Each of these courses of management could potentially influence visual outcome to different extents. However, at the point of analysis, all patients were free of edema both clinically and by SD-OCT exam.

In summary, loss of the foveal IS/OS junction line and loss of inner retinal layers in late stage SD-OCT images in eyes with CRVO significantly correlated with poorer visual outcomes. In addition, the absence of inner retinal layers strongly correlated with the finding macular ischemia by FA. This suggests that SD-OCT can be an important tool both for the evaluation and management of macular edema as well as helping to predict long-term visual prognosis in patients with CRVO, based on these specific structural changes. Judgment as to whether SD-OCT structural predictions are better than current clinical assessments must be reserved pending larger prospective trials.

\section{Acknowledgment}

Tseng Hsiao-Jung, Biostatistical Center for Clinical Research, Chang Gung Memorial Hospital, Taoyuan, Taiwan.

\section{Disclosure}

Dr Richard B Rosen is a member of the Scientific Advisory Boards of OTI-OPKO, OD-OS and Clarity Medical.

\section{References}

1. The Central Vein Occlusion Study Group. Natural history and clinical management of central retinal vein occlusion. Arch Ophthalmol. 1997; 115:486-491.

2. Glacet-Bernard A, Coscas G, Chabanel A, Zourdani A, Lelong F, Samama MM. Prognostic factors for retinal vein occlusion: prospective study of 175 cases. Ophthalmology. 1996;103:551-560.

3. The Central Vein Occlusion Study Group. Evaluation of grid pattern photocoagulation for macular edema in central vein occlusion. M report. Ophthalmology. 1995;102:1425-1433.

4. Binder S, Aggermann T, Brunner S. Long-term effects of radial optic neurotomy for central retinal vein occlusion consecutive interventional case series. Graefes Arch Clin Exp Ophthalmol. 2007;245:1447-1452.

5. Ghazi NG, Noureddine B, Haddad RS, Jurdi FA, Bashshur ZF. Intravitreal tissue plasminogen activator in the management of central retinal vein occlusion. Retina. 2003;23:780-784.

6. Goff MJ, Jumper JM, Yang SS, et al. Intravitreal triamcinolone acetonide treatment of macular edema associated with central retinal vein occlusion. Retina. 2006;26:896-901.

7. Iturralde D, Spaide RF, Meyerle CB, et al. Intravitreal bevacizumab (Avastin) treatment of macular edema in central retinal vein occlusion: a short-term study. Retina. 2006;26:279-284.

8. Pai SA, Shetty R, Vijayan PB, et al. Clinical, anatomic, and electrophysiologic evaluation following intravitreal bevacizumab for macular edema in retinal vein occlusion. Am J Ophthalmol. 2007;143:601-606.

9. Mohamed Q, McIntosh RL, Saw SM, Wong TY. Interventions for central retinal vein occlusion: an evidence-based systematic review. Ophthalmology. 2007;114:507-519.

10. Ota M, Tsujikawa A, Kita M, et al. Integrity of foveal photoreceptor layer in central retinal vein occlusion. Retina. 2008;28:1502-1508.

11. Huang D, Swanson EA, Lin CP, et al. Optical coherence tomography. Science. 1991;254:1178-1181.

12. Hee MR, Izatt JA, Swanson EA, et al. Optical coherence tomography of the human retina. Arch Ophthalmol. 1995;113:325-332.

13. Puliafito CA, Hee MR, Lin CP, et al. Imaging of macular diseases with optical coherence tomography. Ophthalmology. 1995;102:217-229.

14. Drexler W. Ultrahigh-resolution optical coherence tomography. J Biomed Opt. 2004;9:47-74.

15. Nassif N, Cense B, Park BH, et al. In vivo human retinal imaging by ultrahigh-speed spectral domain optical coherence tomography. Opt Lett. 2004;29:480-482.

16. Podoleanu AG, Rosen RB. Combinations of techniques in imaging the retina with high resolution. Prog Retin Eye Res. 2008;27:464-499.

17. Srinivasan VJ, Wojtkowski M, Witkin AJ, et al. High-definition and 3-dimensional imaging of macular pathologies with high-speed ultrahigh-resolution optical coherence tomography. Ophthalmology. 2006;113:2054-2065.

18. Sakamoto A, Hangai M, Yoshimura N. Spectral-domain optical coherence tomography with multiple B-scan averaging for enhanced imaging of retinal diseases. Ophthalmology. 2008;115:1071-1078.

19. Murakami T, Tsujikawa A, Ohta M, et al. Photoreceptor status after resolved macular edema in branch retinal vein occlusion treated with tissue plasminogen activator. Am J Ophthalmol. 2007;143:171-173.

20. Ota M, Tsujikawa A, Murakami T, et al. Association between integrity of foveal photoreceptor layer and visual acuity in branch retinal vein occlusion. Br J Ophthalmol. 2007;91:1644-1649.

21. Ota M, Tsujikawa A, Murakami T, et al. Foveal photoreceptor layer in eyes with persistent cystoid macular edema associated with branch retinal vein occlusion. Am J Ophthalmol. 2008;145:273-280.

22. Chung EJ, Roh MI, Kwon OW, Koh HJ. Effects of macular ischemia on the outcome of intravitreal bevacizumab therapy for diabetic macular edema. Retina. 2008;28:957-963.

23. Scott IU, VanVeldhuisen PC, Oden NL, et al. SCORE Study Investigator Group. SCORE Study report 1: baseline associations between central retinal thickness and visual acuity in patients with retinal vein occlusion. Ophthalmology. 2009;116:504-512. 
24. Tso MO. Pathology of cystoid macular edema. Ophthalmology. 1982;89:902-915.

25. Bowers DK, Finkelstein D, Wolff SM, Green WR. Branch retinal vein occlusion. A clinicopathologic case report. Retina. 1987;7:252-259.

26. Frangieh GT, Green WR, Barraquer-Somers E, Finkelstein D. Histopathologic study of nine branch retinal vein occlusions. Arch Ophthalmol. 1982;100:1132-1140.
27. Green WR, Chan CC, Hutchins GM, Terry JM. Central retinal vein occlusion: a prospective histopathologic study of 29 eyes in 28 cases. Retina. 1981;1:27-55.

28. The Central Vein Occlusion Study Group. Baseline and early natural history report: the Central Vein Occlusion Study. Arch Ophthalmol. 1993;111:1087-1095.

\section{Publish your work in this journal}

Clinical Ophthalmology is an international, peer-reviewed journal covering all subspecialties within ophthalmology. Key topics include: Optometry; Visual science; Pharmacology and drug therapy in eye diseases; Basic Sciences; Primary and Secondary eye care; Patien Safety and Quality of Care Improvements. This journal is indexed on

Submit your manuscript here: http://www.dovepress.com/clinical-ophthalmology-journal

\section{Dovepress}

PubMed Central and CAS, and is the official journal of The Society of Clinical Ophthalmology (SCO). The manuscript management system is completely online and includes a very quick and fair peer-review system, which is all easy to use. Visit http://www.dovepress.com/ testimonials.php to read real quotes from published authors. 